\title{
Some subordination and superordination results of generalized Srivastava-Attiya operator
}

Mohamed Kamal Aouf, Adela Osman Mostafa, Awatif Mohamed Shahin and Samar Mohamed Madian*

* Correspondence:

samar_math@yahoo.com

Department of Mathematics,

Faculty of Science, Mansoura

University, Mansoura 35516, Egypt

\section{Abstract}

In this article, we obtain some subordination and superordination-preserving results of the generalized Srivastava-Attyia operator. Sandwich-type result is also obtained. Mathematics Subject Classification 2000: 30C45.

Keywords: analytic function, Hadamard product, differential subordination, superordination

\section{Introduction}

Let $H(U)$ be the class of functions analytic in $U=\{z \in \mathbb{C}:|z|<1\}$ and $H[a, n]$ be the subclass of $H(U)$ consisting of functions of the form $f(z)=a+a_{n} z^{n}+a_{n+1} z^{\mathrm{n}+1}+\ldots$, with $H_{0}=H[0,1]$ and $H=H[1,1]$. Denote $A(p)$ by the class of all analytic functions of the form

$$
f(z)=z^{p}+\sum_{n=1}^{\infty} a_{p+n} z^{p+n}(p \in \mathbb{N}=\{1,2,3, \ldots\} ; z \in U)
$$

and let $A(1)=A$. For $f, F \in H(U)$, the function $f(z)$ is said to be subordinate to $F(z)$, or $F(z)$ is superordinate to $f(z)$, if there exists a function $\omega(z)$ analytic in $U$ with $\omega(0)=$ 0 and $|\omega(z)|<1(z \in U)$, such that $f(z)=F(\omega(z))$. In such a case we write $f(z) \prec F(z)$. If $F$ is univalent, then $f(z) \prec F(z)$ if and only if $f(0)=F(0)$ and $f(U) \subset F(U)$ (see [1,2]).

Let $\phi: \mathbb{C}^{2} \times U \rightarrow \mathbb{C}$ and $h(z)$ be univalent in $U$. If $p(z)$ is analytic in $U$ and satisfies the first order differential subordination:

$$
\phi\left(p(z), z p^{\prime}(z) ; z\right) \prec h(z),
$$

then $p(z)$ is a solution of the differential subordination (1.2). The univalent function $q(z)$ is called a dominant of the solutions of the differential subordination (1.2) if $p(z)$ $\prec q(z)$ for all $p(z)$ satisfying (1.2). A univalent dominant $\tilde{q}$ that satisfies $\tilde{q} \prec q$ for all dominants of (1.2) is called the best dominant. If $p(z)$ and $\varphi\left(p(z), z p^{\prime}(z) ; z\right)$ are univalent in $U$ and if $p(z)$ satisfies the first order differential superordination:

$$
h(z) \prec \phi\left(p(z), z p^{\prime}(z) ; z\right),
$$

then $p(z)$ is a solution of the differential superordination (1.3). An analytic function $q$ $(z)$ is called a subordinant of the solutions of the differential superordination (1.3) if $q$ 
$(z) \prec p(z)$ for all $p(z)$ satisfying (1.3). A univalent subordinant $\tilde{q}$ that satisfies $q \prec \tilde{q}$ for all subordinants of $(1.3)$ is called the best subordinant (see [1,2]).

The general Hurwitz-Lerch Zeta function $\Phi(z, s, a)$ is defined by:

$$
\Phi(z, s, a)=\sum_{n=0}^{\infty} \frac{z^{n}}{(n+a)^{s}}
$$

$\left(a \in \mathbb{C} \backslash \mathbb{Z}_{0}^{-} ; \mathbb{Z}_{0}^{-}=\{0,-1,-2, \ldots\} ; s \in \mathbb{C}\right.$ when $|z|<1 ; R\{s\}>1$ when $\left.|z|=1\right)$.

For further interesting properties and characteristics of the Hurwitz-Lerch Zeta function $\Phi(z, s, a)$ (see [3-7]).

Recently, Srivastava and Attiya [8] introduced the linear operator $L_{s, b}: A \rightarrow A$, defined in terms of the Hadamard product by

$$
L_{s, b}(f)(z)=G_{s, b}(z) * f(z)\left(z \in U ; b \in \mathbb{C} \backslash \mathbb{Z}_{0}^{-} ; s \in \mathbb{C}\right),
$$

where

$$
G_{s, b}=(1+b)^{s}\left[\Phi(z, s, b)-b^{-s}\right](z \in U) .
$$

The Srivastava-Attiya operator $L_{s, b}$ contains among its special cases, the integral operators introduced and investigated by Alexander [9], Libera [10] and Jung et al. [11].

Analogous to $L_{s, b}$, Liu [12] defined the operator $J_{p, s, b}: A(p) \rightarrow A(p)$ by

$$
J_{p, s, b}(f)(z)=G_{p, s, b}(z) * f(z)\left(z \in U ; b \in \mathbb{C} / \mathbb{Z}_{0}^{-} ; s \in \mathbb{C} ; p \in \mathbb{N}\right),
$$

where

$$
G_{p, s, b}=(1+b)^{s}\left[\Phi_{p}(z, s, b)-b^{-s}\right]
$$

and

$$
\Phi_{p}(z, s, b)=\frac{1}{b^{s}}+\sum_{n=0}^{\infty} \frac{z^{n+p}}{(n+1+b)^{s}} .
$$

It is easy to observe from (1.7) and (1.8) that

$$
J_{p, s, b}(f)(z)=z^{p}+\sum_{n=1}^{\infty}\left(\frac{1+b}{n+1+b}\right)^{s} a_{n+p} z^{n+p} .
$$

We note that

(i) $J_{p, 0, b}(f)(z)=f(z)$;

(ii) $J_{1, s, b}(f)(z)=L_{s, b} f(z)\left(s \in \mathbb{C}, b \in \mathbb{C} \backslash \mathbb{Z}_{0}^{-}\right)$, where the operator $L_{s, b}$ was introduced by Srivastava and Attiya [8];

(iii) $J_{p, 1, v+p-1}(f)(z)=F_{v, p}(f(z))(v>-p, p \in \mathbb{N})$, where the operator $F_{v, p}$ was introduced by Choi et al. [13];

(iv) $J_{p, \alpha, p}(f)(z)=I_{p}^{\alpha} f(z)(\alpha \geq 0, p \in \mathbb{N})$, where the operator $I_{p}^{\alpha}$ was introduced by Shams et al. [14];

(v) $J_{p, m, p-1}(f)(z)=J_{p}^{m} f(z)\left(m \in \mathbb{N}_{0}=\mathbb{N} \cup\{0\}, p \in \mathbb{N}\right)$, where the operator $J_{p}^{m}$ was introduced by El-Ashwah and Aouf [15]; 
(vi) $J_{p, m, p+l-1}(f)(z)=J_{p}^{m}(l) f(z)\left(m \in \mathbb{N}_{0}, p \in \mathbb{N}, l \geq 0\right)$, where the operator $J_{p}^{m}(l)$ was introduced by El-Ashwah and Aouf [15].

It follows from (1.9) that:

$$
z\left(J_{p, s+1, b}(f)(z)\right)^{\prime}=(b+1) J_{p, s, b}(f)(z)-(b+1-p) J_{p, s+1, b}(f)(z) .
$$

To prove our results, we need the following definitions and lemmas.

Definition 1 [1]Denote by $\mathcal{F}$ the set of all functions $q(z)$ that are analytic and injective on $\bar{U} \backslash E(q)$ where

$$
E(q)=\left\{\zeta \in \partial U: \lim _{z \rightarrow \zeta} q(z)=\infty\right\}
$$

and are such that $q^{\prime}(\zeta) \neq 0$ for $\zeta \in \delta U \backslash E(q)$. Further let the subclass of $\mathcal{F}$ for which $q$ $(0)=a$ be denoted by $\mathcal{F}(a), \mathcal{F}(0) \equiv \mathcal{F}_{0}$ and $\mathcal{F}(1) \equiv \mathcal{F}_{1}$.

Definition 2 [2]A function $L(z, t)(z \in U, t \geq 0)$ is said to be a subordination chain if $L(0, t)$ is analytic and univalent in $U$ for all $t \geq 0, L(z, 0)$ is continuously differentiable on $[0 ; 1)$ for all $z \in U$ and $L\left(z, t_{1}\right) \prec L\left(z, t_{2}\right)$ for all $0 \leq t_{1} \leq t_{2}$.

Lemma $1[16]$ The function $L(z, t): U \times[0 ; 1) \rightarrow \mathbb{C}$ of the form

$$
L(z, t)=a_{1}(t) z+a_{2}(t) z^{2}+\cdots\left(a_{1}(t) \neq 0 ; t \geq 0\right)
$$

and $\lim _{t \rightarrow \infty}\left|a_{1}(t)\right|=\infty$ is a subordination chain if and only if

$$
\operatorname{Re}\left\{\frac{z \partial L(z, t) / \partial z}{\partial L(z, t) / \partial t}\right\}>0(z \in U, t \geq 0) .
$$

Lemma 2 [17] Suppose that the function $\mathcal{H}: \mathbb{C}^{2} \rightarrow \mathbb{C}$ satisfies the condition

$\operatorname{Re}\{\mathcal{H}(i s ; t)\} \leq 0$

for all real $s$ and for all $t \leq-n\left(1+s^{2}\right) / 2, n \in \mathbb{N}$. If the function $p(z)=1+p_{n} z^{n}+p_{n}$ ${ }_{+1} \mathrm{z}^{\mathrm{n}+1}+\ldots$ is analytic in $U$ and

$$
\operatorname{Re}\left\{\mathcal{H}\left(p(z) ; z p^{\prime}(z)\right)\right\}>0(z \in U),
$$

then $\operatorname{Re}\{p(z)\}>0$ for $z \in U$.

Lemma $3[18]$ Let $\kappa, \gamma \in \mathbb{C}$ with $\kappa \neq 0$ and let $h \in H(U)$ with $h(0)=c$. If $\operatorname{Re}\{\kappa h(z)+$ $\gamma_{\}}>0(z \in U)$, then the solution of the following differential equation:

$$
q(z)+\frac{z q^{\prime}(z)}{\kappa q(z)+\gamma}=h(z)(z \in U ; q(0)=c)
$$

is analytic in $U$ and satisfies $\operatorname{Re}\{\kappa q(z)+\gamma\}>0$ for $z \in U$.

Lemma 4 [1] Let $p \in \mathcal{F}(a)$ and let $q(z)=a+a_{n} z^{n}+a_{\mathrm{n}+1} \mathrm{z}^{\mathrm{n}+1}+$...be analytic in $U$ with $q(z) \neq a$ and $n \geq 1$. If $q$ is not subordinate to $p$, then there exists two points $z_{0}=$ $r_{0} e^{i \theta} \in U$ and $\zeta_{0} \in \delta U \backslash E(q)$ such that

$$
q\left(U_{r_{0}}\right) \subset p(U) ; q\left(z_{0}\right)=p\left(\zeta_{0}\right) \text { and } z_{0} p^{\prime}\left(z_{0}\right)=m \zeta_{0} p^{\prime}\left(\zeta_{0}\right)(m \geq n) .
$$

Lemma $5[2]$ Let $q \in H[a ; 1]$ and $\phi: \mathbb{C}^{2} \rightarrow \mathbb{C}$. Also set $\phi\left(q(z), z q^{\prime}(z)\right)=h(z)$. If $L(z, t)=$ $\phi\left(q(z), t z q^{\prime}(z)\right)$ is a subordination chain and $q \in H[a ; 1] \cap \mathcal{F}(a)$, then 


$$
h(z) \prec \varphi\left(q(z), z q^{\prime}(z)\right),
$$

implies that $q(z) \prec p(z)$. Furthermore, if $\phi\left(q(z), z q^{\prime}(z)\right)=h(z)$ has a univalent solution $q \in \mathcal{F}(a)$, then $q$ is the best subordinant.

In the present article, we aim to prove some subordination-preserving and superordination-preserving properties associated with the integral operator $J_{p, s, b}$. Sandwich-type result involving this operator is also derived.

\section{Main results}

Unless otherwise mentioned, we assume throughout this section that $b \in \mathbb{C} \backslash \mathbb{Z}_{0}^{-}, s \in \mathbb{C}$, $\operatorname{Re}\{b\}, \mu>0, p \in \mathbb{N}, z \in \mathbb{U}$ and the powers are understood as principle values.

Theorem 1. Let $f, g \in A(p)$ and

$$
\operatorname{Re}\left\{1+\frac{z \phi^{\prime \prime}(z)}{\phi^{\prime}(z)}\right\}>-\delta\left(\phi(z)=\left(\frac{J_{p, s-1, b}(g)(z)}{J_{p, s, b}(g)(z)}\right)\left(\frac{J_{p, s, b}(g)(z)}{z^{p}}\right)^{\mu} ; z \in U\right),
$$

where $\delta$ is given by

$$
\delta=\frac{1+\mu^{2}|b+1|^{2}-\left|1-\mu^{2}(b+1)^{2}\right|}{4 \mu[1+\operatorname{Re}\{b\}]}(z \in U) .
$$

Then the subordination condition

$$
\left(\frac{J_{p, s-1, b}(f)(z)}{J_{p, s, b}(f)(z)}\right)\left(\frac{J_{p, s, b}(f)(z)}{z^{p}}\right)^{\mu} \prec\left(\frac{J_{p, s-1, b}(g)(z)}{J_{p, s, b}(g)(z)}\right)\left(\frac{J_{p, s, b}(g)(z)}{z^{p}}\right)^{\mu},
$$

implies that

$$
\left(\frac{J_{p, s, b}(f)(z)}{z^{p}}\right)^{\mu} \prec\left(\frac{J_{p, s, b}(g)(z)}{z^{p}}\right)^{\mu}
$$

where $\left(\frac{J_{p, s, b}(g)(z)}{z^{p}}\right)^{\mu}$ is the best dominant.

Proof. Let us define the functions $F(z)$ and $G(z)$ in $U$ by

$$
F(z)=\left(\frac{J_{p, s, b}(f)(z)}{z^{p}}\right)^{\mu} \text { and } G(z)=\left(\frac{J_{p, s, b}(g)(z)}{z^{p}}\right)^{\mu}(z \in U)
$$

and without loss of generality we assume that $G(z)$ is analytic, univalent on $\bar{U}$ and

$$
G^{\prime}(\zeta) \neq 0(|\zeta|=1)
$$

If not, then we replace $F(z)$ and $G(z)$ by $F(\rho z)$ and $G(\rho z)$, respectively, with $0<\rho<1$. These new functions have the desired properties on $\bar{U}$, so we can use them in the proof of our result and the results would follow by letting $\rho \rightarrow 1$.

We first show that, if

$$
q(z)=1+\frac{z G^{\prime \prime}(z)}{G^{\prime}(z)}(z \in U)
$$

then

$$
\operatorname{Re}\{q(z)\}>0(z \in U) .
$$


From (1.10) and the definition of the functions $G, \varphi$, we obtain that

$$
\phi(z)=G(z)+\frac{z G^{\prime}(z)}{\mu(b+1)} .
$$

Differentiating both sides of (2.7) with respect to $z$ yields

$$
\phi^{\prime}(z)=\left(1+\frac{1}{\mu(b+1)}\right) G^{\prime}(z)+\frac{z G^{\prime \prime}(z)}{\mu(b+1)} .
$$

Combining (2.6) and (2.8), we easily get

$$
1+\frac{z \phi^{\prime \prime}(z)}{\phi^{\prime}(z)}=q(z)+\frac{z q^{\prime}(z)}{q(z)+\mu(b+1)}=h(z)(z \in U) .
$$

It follows from (2.1) and (2.9) that

$$
\operatorname{Re}\{h(z)+\mu(b+1)\}>0(z \in U)
$$

Moreover, by using Lemma 3, we conclude that the differential Equation (2.9) has a solution $q(z) \in H(U)$ with $h(0)=q(0)=1$. Let

$$
\mathcal{H}(u, v)=u+\frac{v}{u+\mu(b+1)}+\delta,
$$

Where $\delta$ is given by (2.2). From (2.9) and (2.10), we obtain $\left.\operatorname{Re}\left\{\mathcal{H}(q(z) ;) z q^{\prime}(z)\right)\right\}>0(z \in U)$.

To verify the condition

$$
\operatorname{Re}\{\mathcal{H}(i \vartheta ; t)\} \leq 0\left(\vartheta \in \mathbb{R} ; t \leq-\frac{1+\vartheta^{2}}{2}\right)
$$

we proceed as follows:

$$
\begin{aligned}
\operatorname{Re}\{\mathcal{H}(i \vartheta ; t)\} & =\operatorname{Re}\left\{i \vartheta+\frac{t}{\mu(b+1)+i \vartheta}+\delta\right\}=\frac{t \mu(1+\operatorname{Re}(b))}{|\mu(b+1)+i \vartheta|^{2}}+\delta \\
& \leq-\frac{\Upsilon(b, \vartheta, \delta)}{2|\mu(b+1)+i \vartheta|^{2}}
\end{aligned}
$$

where

$$
\Upsilon(b, \vartheta, \delta)=[\mu(1+\operatorname{Re}(b))-2 \delta] \vartheta^{2}-4 \delta \mu \operatorname{Im}(b) \vartheta-2 \delta|\mu(b+1)|^{2}+\mu(1+\operatorname{Re}\{b\}) .
$$

For $\delta$ given by (2.2), the coefficient of $\vartheta^{2}$ in the quadratic expression $\Upsilon(b, \vartheta, \delta)$ given by (2.12) is positive or equal to zero. To check this, put $\mu(b+1)=c$, so that

$$
\mu(1+\operatorname{Re}(b))=c_{1} \text { and } \mu \operatorname{Im}(b)=c_{2} .
$$

We thus have to verify that

$$
c_{1}-2 \delta \geq 0,
$$

or

$$
c_{1} \geq 2 \delta=\frac{1+|c|^{2}-\left|1-c^{2}\right|}{2 c_{1}} .
$$


This inequality will hold true if

$$
2 c_{1}^{2}+\left|1-c^{2}\right| \geq 1+|c|^{2}=1+c_{1}^{2}+c_{2}^{2}
$$

that is, if

$$
\left|1-c^{2}\right| \geq 1-\operatorname{Re}\left(c^{2}\right)
$$

which is obviously true. Moreover, the quadratic expression $\Upsilon(b, \vartheta, \delta)$ by $\vartheta$ in (2.12) is a perfect square for the assumed value of $\delta$ given by (2.2). Hence we see that (2.11) holds. Thus, by Lemma 2, we conclude that

$$
\operatorname{Re}\{q(z)\}>0(z \in U),
$$

that is, that $G$ defined by $(2.5)$ is convex (univalent) in $U$. Next, we prove that the subordination condition (2.3) implies that

$$
F(z) \prec G(z)
$$

for the functions $F$ and $G$ defined by (2.5). Consider the function $L(z, t)$ given by

$$
L(z, t)=G(z)+\frac{(1+t) z G^{\prime}(z)}{\mu(b+1)}(0 \leq t<\infty ; z \in U) .
$$

We note that

$$
\left.\frac{\partial L(z, t)}{\partial z}\right|_{z=0}=G^{\prime}(0)\left(1+\frac{1+t}{\mu(b+1)}\right) \neq 0(0 \leq t<\infty ; z \in U ; \operatorname{Re}\{\mu(b+1)\}>0) .
$$

This show that the function

$$
L(z, t)=a_{1}(t) z+\cdots
$$

satisfies the condition $a_{1}(t) \neq 0(0 \leq t<\infty)$. Further, we have

$$
\operatorname{Re}\left\{\frac{z \partial L(z, t) / \partial z}{\partial L(z, t) / \partial t}\right\}=\operatorname{Re}\{\mu(b+1)+(1+t) q(z)\}>0(0 \leq t<\infty ; z \in U) .
$$

Since $G(z)$ is convex and $\operatorname{Re}\{\mu(b+1)\}>0$. Therefore, by using Lemma 1 , we deduce that $L(z, t)$ is a subordination chain. It follows from the definition of subordination chain that

$$
\phi(z)=G(z)+\frac{z G^{\prime}(z)}{\mu(b+1)}=L(z, 0)
$$

and

$$
L(z, 0) \prec L(z, t)(0 \leq t<\infty)
$$

which implies that

$$
L(\zeta, t) \notin L(U, 0)=\phi(U)(0 \leq t<\infty ; \zeta \in \partial U)
$$

If $F$ is not subordinate to $G$, by using Lemma 4, we know that there exist two points $z_{0} \in U$ and $\zeta_{0} \in \partial U$ such that

$$
F\left(z_{0}\right)=G\left(\zeta_{0}\right) \text { and } z_{0} F^{\prime}\left(z_{0}\right)=(1+t) \zeta_{0} G^{\prime}\left(\zeta_{0}\right)(0 \leq t<\infty) .
$$


Hence, by using (2.5), (2.13), (2.15) and (2.3), we have

$$
\begin{aligned}
L\left(\zeta_{0}, t\right) & =G\left(\zeta_{0}\right)+\frac{(1+t) \zeta_{0} G^{\prime}\left(\zeta_{0}\right)}{\mu(b+1)}=F\left(z_{0}\right)+\frac{z_{0} F^{\prime}\left(z_{0}\right)}{\mu(b+1)} \\
& =\left(\frac{J_{p, s-1, b}(f)(z)}{J_{p, s, b}(f)(z)}\right)\left(\frac{J_{p, s, b}(f)(z)}{z^{p}}\right)^{\mu} \in \phi(U) .
\end{aligned}
$$

This contradicts (2.14). Thus, we deduce that $F \prec G$. Considering $F=G$, we see that the function $G$ is the best dominant. This completes the proof of Theorem 1.

We now derive the following superordination result.

Theorem 2. Let $f, g \in A(p)$ and

$$
\operatorname{Re}\left\{1+\frac{z \phi^{\prime \prime}(z)}{\phi^{\prime}(z)}\right\}>-\delta\left(\phi(z)=\left(\frac{J_{p, s-1, b}(g)(z)}{J_{p, s, b}(g)(z)}\right)\left(\frac{J_{p, s, b}(g)(z)}{z^{p}}\right)^{\mu} ; z \in U\right),
$$

where $\delta$ is given by (2.2). If the function $\left(\frac{J_{p, s-1, b}(f)(z)}{J_{p, s, b}(f)(z)}\right)\left(\frac{J_{p, s, b}(f)(z)}{z^{p}}\right)^{\mu}$ is univalent in $U$ and $\left(\frac{J_{p, s, b}(f)(z)}{z^{p}}\right)^{\mu} \in \mathcal{F}$, then the superordination condition

$$
\left(\frac{J_{p, s-1, b}(g)(z)}{J_{p, s, b}(g)(z)}\right)\left(\frac{J_{p, s, b}(g)(z)}{z^{p}}\right)^{\mu} \prec\left(\frac{J_{p, s-1, b}(f)(z)}{J_{p, s, b}(f)(z)}\right)\left(\frac{J_{p, s, b}(f)(z)}{z^{p}}\right)^{\mu},
$$

implies that

$$
\left(\frac{J_{p, s, b}(g)(z)}{z^{p}}\right)^{\mu} \prec\left(\frac{J_{p, s, b}(f)(z)}{z^{p}}\right)^{\mu}
$$

where $\left(\frac{J_{p, s, b}(f)(z)}{z^{p}}\right)^{\mu}$ is the best subordinant.

Proof. Suppose that the functions $F, G$ and $q$ are defined by (2.5) and (2.6), respectively. By applying similar method as in the proof of Theorem 1 , we get

$$
\operatorname{Re}\{q(z)\}>0(z \in U) .
$$

Next, to arrive at our desired result, we show that $G \prec F$. For this, we suppose that the function $L(z, t)$ be defined by (2.13). Since $G$ is convex, by applying a similar method as in Theorem 1, we deduce that $L(z, t)$ is subordination chain. Therefore, by using Lemma 5, we conclude that $G \prec F$. Moreover, since the differential equation

$$
\phi(z)=G(z)+\frac{z G^{\prime}(z)}{\mu(b+1)}=\varphi\left(G(z), z G^{\prime}(z)\right)
$$

has a univalent solution $G$, it is the best subordinant. This completes the proof of Theorem 2.

Combining the above-mentioned subordination and superordination results involving the operator $J_{p, s, b}$, the following "sandwich-type result" is derived.

Theorem 3. Let $f, g_{j} \in A(p)(j=1,2)$ and

$$
\operatorname{Re}\left\{1+\frac{z \phi_{j}^{\prime \prime}(z)}{\phi_{j}^{\prime}(z)}\right\}>-\delta\left(\phi_{j}(z)=\left(\frac{J_{p, s-1, b}\left(g_{j}\right)(z)}{J_{p, s, b}\left(g_{j}\right)(z)}\right)\left(\frac{J_{p, s, b}\left(g_{j}\right)(z)}{z^{p}}\right)^{\mu}(j=1,2) ; z \in U\right),
$$


where $\delta$ is given by (2.2). If the function $\left(\frac{J_{p, s-1, b}(f)(z)}{J_{p, s, b}(f)(z)}\right)\left(\frac{J_{p, s, b}(f)(z)}{z^{p}}\right)^{\mu}$ is univalent in $U$ and $\left(\frac{J_{p, s, b}(f)(z)}{z^{p}}\right)^{\mu} \in \mathcal{F}$, then the condition

$$
\begin{aligned}
\left(\frac{J_{p, s-1, b}\left(g_{1}\right)(z)}{J_{p, s, b}\left(g_{1}\right)(z)}\right)\left(\frac{J_{p, s, b}\left(g_{1}\right)(z)}{z^{p}}\right)^{\mu} & \prec\left(\frac{J_{p, s-1, b}(f)(z)}{J_{p, s, b}(f)(z)}\right)\left(\frac{J_{p, s, b}(f)(z)}{z^{p}}\right)^{\mu} \\
& \prec\left(\frac{J_{p, s-1, b}\left(g_{2}\right)(z)}{J_{p, s, b}\left(g_{2}\right)(z)}\right)\left(\frac{J_{p, s, b}\left(g_{2}\right)(z)}{z^{p}}\right)^{\mu},
\end{aligned}
$$

implies that

$$
\left(\frac{J_{p, s, b}\left(g_{1}\right)(z)}{z^{p}}\right)^{\mu} \prec\left(\frac{J_{p, s, b}(f)(z)}{z^{p}}\right)^{\mu} \prec\left(\frac{J_{p, s, b}\left(g_{2}\right)(z)}{z^{p}}\right)^{\mu},
$$

where $\left(\frac{J_{p, s, b}\left(g_{1}\right)(z)}{z^{p}}\right)^{\mu}$ and $\left(\frac{J_{p, s, b}\left(g_{2}\right)(z)}{z^{p}}\right)^{\mu}$ are, respectively, the best subordinant and the best dominant.

Remark. (i) Putting $\mu=1, b=p$ and $s=\alpha(\alpha=0, p \in \mathbb{N})$ in our results of this article, we obtain the results obtained by Aouf and Seoudy [19];

(ii) Specializing the parameters $s$ and $b$ in our results of this article, we obtain the results for the corresponding operators $F_{v, p}, I_{p}^{\alpha}, J_{p}^{m}$ and $J_{p}^{m}(l)$ which are defined in the introduction.

\section{Authors' contributions}

All authors read and approved the final manuscript.

\section{Competing interests}

The authors declare that they have no competing interests.

Received: 8 December 2011 Accepted: 24 May 2012 Published: 24 May 2012

\section{References}

1. Miller, SS, Mocanu, PT: Differential Subordinations: Theory and Applications, Series on Monographs and Textbooks in Pure and Applied Mathematics. Marcel Dekker, New York/Basel225 (2000)

2. Miller, S, Mocanu, PT: Subordinants of differential superordinations. Complex Var Theory Appl. 48(10), 815-826 (2003). doi:10.1080/02781070310001599322

3. Choi, JH, Srivastava, HM: Certain families of series associated with the Hurwitz-Lerch Zeta function. Appl Math Comput. 170, 399-409 (2005). doi:10.1016/j.amc.2004.12.004

4. Lin, S-D, Srivastava, HM: Some families of the Hurwitz-Lerch Zeta functions and associated fractional derivative and other integral representations. Appl Math Comput. 154, 725-733 (2004). doi:10.1016/S0096-3003(03)00746-X

5. Lin, S-D, Srivastava, HM, Wang, P-Y: Some expansion formulas for a class of generalized Hurwitz-Lerch Zeta functions. Integr Trans Spec Funct. 17, 817-827 (2006). doi:10.1080/10652460600926923

6. Luo, Q-M, Srivastava, HM: Some generalizations of the Apostol-Bernoulli and Apostol-Euler polynomials. J Math Anal Appl. 308, 290-302 (2005). doi:10.1016/j.jmaa.2005.01.020

7. Srivastava, HM, Choi, J: Series Associated with the Zeta and Related Functions. Kluwer Academic Publishers, Dordrecht (2001)

8. Srivastava, HM, Attiya, AA: An integral operator associated with the Hurwitz-Lerch Zeta function and differential subordination. Integr Trans Spec Funct. 18, 207-216 (2007). doi:10.1080/10652460701208577

9. Alexander, JW: Functions which map the interior of the unit circle upon simple regions. Ann Math Ser. 17(2), 12-22 (1915)

10. Libera, RJ: Some classes of regular univalent functions. Proc Am Math Soc. 16, 755-758 (1969)

11. Jung, TB, Kim, YC, Srivastava, HM: The Hardy space of analytic functions associated with certain one-parameter families of integral operators. J Math Anal Appl. 176, 138-147 (1993). doi:10.1006/jmaa.1993.1204

12. Liu, J-L: Subordinations for certain multivalent analytic functions associated with the generalized Srivastava-Attiya operator. Integr Trans Spec Funct. 18, 207-216 (2007). doi:10.1080/10652460701208577

13. Choi, JH, Saigo, M, Srivastava, HM: Some inclusion properties of a certain family of integral operators. J Math Anal Appl. 276, 432-445 (2002). doi:10.1016/S0022-247X(02)00500-0 
14. Shams, S, Kulkarni, SR, Jahangiri, JM: Subordination properties of $p$-valent functions defined by integral operators. Int J Math Math Sci 2006, 1 (2006). 3 (Article ID 94572)

15. El-Ashwah, RM, Aouf, MK: Some properties of new integral operator. Acta Univ Apulensis. 24, 51-61 (2010) (2010)

16. Pommerenke, Ch: Univalent Functions. Vandenhoeck and Ruprecht, Göttingen. (1975)

17. Miller, SS, Mocanu, PT: Differential subordinations and univalent functions. Michigan Math J. 28(2), 157-172 (1981)

18. Miller, SS, Mocanu, PT: Univalent solutions of Briot-Bouquet differential equations. J Diff Equ. 56(3), 297-309 (1985). doi:10.1016/0022-0396(85)90082-8

19. Aouf, MK, Seoudy, TM: Some preserving subordination and superordination results of certain integral operator. Int J Open Probl Complex Analysis. 3(3), 1-8 (2011)

doi:10.1186/1029-242X-2012-115

Cite this article as: Aouf et al:: Some subordination and superordination results of generalized Srivastava-Attiya operator. Journal of Inequalities and Applications 2012 2012:115.

\section{Submit your manuscript to a SpringerOpen ${ }^{\circ}$} journal and benefit from:

- Convenient online submission

- Rigorous peer review

- Immediate publication on acceptance

- Open access: articles freely available online

- High visibility within the field

- Retaining the copyright to your article

Submit your next manuscript at $\gg$ springeropen.com 